\title{
DigiAID: A Wearable Health Platform for Automated Self-tagging in Emergency Cases
}

\author{
Abdelmajid Khelil ${ }^{1}$, Faisal K. Shaikh ${ }^{1,2}$, Adil A. Sheikh ${ }^{1}$, Emad Felemban ${ }^{1}$, and Hattan Bojan ${ }^{1}$ \\ ${ }^{1}$ Science \& Technology Unit \\ Umm Al-Qura University, Makkah, KSA \\ ${ }^{2}$ Mehran University of Engineering and Technology, Jamshoro, Pakistan.
}

\begin{abstract}
The presence medicine usually leads to explosive costs for a full medical coverage in particular for rural areas, frequent nomadic patients and mass casualty incidents. Therefore, advanced tele-medicine such as mobile health (m-Health) is gaining at importance. Body Sensor Networks (BSN) represent a fundamental tool to enable future tele-medicine. In this paper, we present a novel experimental BSN platform that allows to (a) measure important health indications, and (b) to map the health status of the individual carrying the platform into a set of predefined classes. We describe the components of the experimental platform while highlighting its key benefits to the research community through a set of experiments.
\end{abstract}

Key words: Body Sensor Networks; Mobile Health; Emergency Rescue; First Response

\section{INTRODUCTION}

Electronic health (e-Health) and mobile health (m-Health) are increasingly considered to be key drivers for the progress of health systems. They are not less relevant than the development of new medication or treatment processes. Unfortunately, there is still only little work for supporting first aid in rural areas or in mass casualties scenarios, though these scenarios are worldwide one of the most cover demanding health disciplines. The current social, economical and ecological trends of massive urbanization and climate changes impose painful changes to the healthcare landscape and further complicate the emergency support in rural and mass casualty areas. The massive urbanization moves the focus of healthcare to urban areas and therefore hardens the healthcare capabilities in rural areas. The climate changes increase the number of disasters and the number of casualties involved putting more challenges on first responders.

Fortunately, big advances have been made towards smart living and health monitoring systems that support telemedicine. In particular, Body Sensor Networks (BSN) [1] and wireless communications [2] represent powerful techniques for significantly advancing healthcare in emergency scenarios and tele-medicine for rural areas. This paper demonstrates the feasibility of using cost-effective BSNs to address critical difficulties of current emergency response process. We propose Digital first Aid box (DigiAID), a BSN platform that can be worn by patients (out of direct medical coverage) so that they can compute and transfer the level of their urgency according to the national or international rating system. Without loss of generality, we illustrate the operation of DigiAID using the established system of North America, i.e., START system (Simple Triage and Rapid Treatment) [3]. DigiAID is designed to support the first responders to proactively well plan the rescue operation according to local observations gathered through the worn BSNs. This makes it possible to conduct the patient classification and ranking without first responders and even to automate this tedious but fundamental pre-rescue phase.

In a prior work [4], we were the first to develop a set of algorithms to calculate the urgency class of a patient through measuring three basic health indications. In this paper, we propose a real implementation of these algorithms on a commercial-off-the-shelf (COTS) low-cost BSN platform. In addition, we present some experimental results that we conducted using this platform to validate our algorithms. This platform builds the basis for supporting the triage and ranking of patients in mass casualty scenarios as proposed in [5].

The key contribution of this paper is to design a real experimental BSN platform that allows to validate automated self-tagging and ranking algorithms and enable fieldtest for emergency health support. The core design requirements are resilience to sensing failures, efficiency with respect to limited BSN resources such as energy and processing capabilities.

The remainder of this paper is structured as follows. In Sec. II, we propose our BSN platform to support automated self-tagging of patients in emergency cases. In Sec. III, we evaluate the performance of our algorithms on the proposed experimental platform.

\section{OUR DIGIAID FrAMEWORK AND EXPERIMENTAL PLATFORM}

First, we present our methodology. Next, we briefly summarize our prior work on automated self-tagging algorithms. Then, we present the details of our experimental platform.

\section{A. Methodology}

Inspired by first aid box in buildings, cars, etc and life vests in airplanes, our innovation is to build a set of new digital first aid tools using health BSNs for health monitoring using reasonable-cost sensors such as ECG, PCG, EDG, pressure, pulse, temperature, oximeter, etc.

DigiAID is a tool that we propose to bring rapid assistance to individual patients or communities. The rapidity is ensured through an early diagnosis and assessment of the health condition with an expert knowledge. All what we require is a body sensor network that can measure important health indications in real-time and then be able to judge on the criticality of the patient. Our proposal is therefore, the automation of the first 

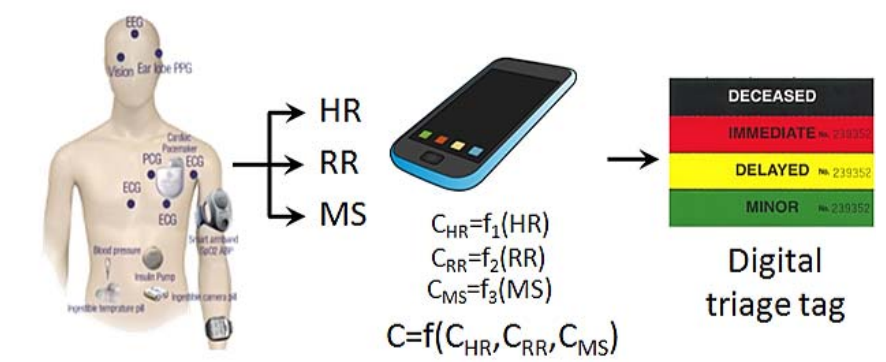

Body
Instrumentation

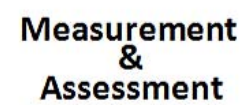

Tagging

Fig. 1: DigiAID operation

responders tasks. This results in more rapid and cost-efficient rescue operation. Thus allowing to save lifes and to avoid under- and over-provisioning of rescue efforts.

We propose DigiAID may be carried in the pocket or placed in the car, in the office, at home, etc to be ready to be worn easily and immediately when somebody suspects a degradation in his/her health conditions.

In Fig. 1, we illustrate the operation of the DigiAID. This operation is tightly tightened to the START system. However, it may easily modified to accommodate national specificities, where needed.

\section{B. Proposed Algorithms for Automated Self-Tagging}

In [4], we proposed three approaches to achieve an intelligent tagging system with much better timeliness and accuracy performance comparable to START. The proposed approaches are (i) table-based, (ii) aggregation-based, and (iii) Fuzzy-Logic-based tagging. The table-based tagging is like the START system but is automated and allows a much higher tagging granularity through supporting 19 classes instead of 4 classes. Unfortunately, table-based approaches are hardly tunable as they can not be easily adapted to specific region and national policies. For fine-grained and tunable tagging, we defined (1) continuous value ranges for criticality $C$, i.e., in the interval [0,1], and (2) three elementary criticality functions $C_{R R}, C_{H R}$, and $C_{M S}$, where each calculates a continuous elementary criticality value for one vital sign Respiratory Rate $(R R)$, Heart Rate $(H R)$ and Mental Status $(M S)$, respectively. Accordingly, we design two other advanced approaches to calculate an overall criticality level $C$, i.e., the aggregationbased and the Fuzzy-Logic-based approaches. For tagging, we provided a flexible mapping process to determine the tagging class depending on the computed criticality value $C$. Due to space limitation, we abstain from the details of the algorithms, which can be found in [4].

\section{Experimental Platform}

In this section, we elaborate our hardware choices and on how we implemented the proposed algorithms in order to evaluate their performance in more realistic conditions.
1) Hardware Platform Selection: Several experimental BSN platforms exist such as [6] [7] [8]. Only a subset of them enable biometric and medical applications [9] [10] [11] [12] [13] [14], etc. The core functionality provided by these platforms is to monitor the Human body in real time by capturing some of its key health state indicators and to collect sensitive data for subsequent analysis and medical diagnosis. In the following, we briefly analyse in order to select the most suitable one for implementing our targeted application.

Several academic platforms such as [11] [12] [15], MobiHealth [16] [17], MYOTEL [14], Personal Health Monitor (PHM) [13] [18] have been developed and applied in various clinical settings. However, these platforms are still unavailable commercially and therefore can not be used for our purposes.

On the other side, more and more commercial platforms are being released. Examples include the Shimmer Health BSN [9] [19] from Intel, and the Simband health sensor platform [20] from Samsung. Unfortunately, these platforms still are expensive and require proprietary hardware and software components.

The e-Health Sensor Shield [10] from Lebelium Cooking Hacks relies on two popular sensing and computing platforms, i.e., Arduino and Raspberry Pi [21]. Nine different sensors are available: Pulse, blood pressure, oxygen in blood (SPO2), electrocardiogram (EKG), airflow, glucometer, galvanic skin response (GSR), patient position and body temperature. Extracted health information can then be wirelessly transmitted using six varied connectivity options, i.e., WiFi, 3G, GPRS, Bluetooth, 802.15.4 and ZigBee, depending on the application.

For the implementation of DigiAID, we selected the eHealth Sensor Shield [10]. Being HW/SW-open-source and low-cost, the platform promises a faster acceptance and spread of our implementation, as well as large-scale trials in mass casualty incidents.

2) Hardware Components: The DigiAID platform uses COTS hardware components. DigiAID consists of one Raspberry-Pi board augmented with the Cooking Hacks eHealth shield. It also uses a few medical sensors, keypad, GPS and graphical LCD for user interaction. There are primarily three sensors attached to the DigiAID, i.e., airflow sensor, pulse oximeter and position sensor, which are available with the e-Health kit. The airflow sensor is used to check the Respiratory Rate $(R R)$ of the patient. The pulse oximeter is a noninvasive method of indicating the arterial oxygen saturation of functional hemoglobin. It is very useful in any setting where the patients oxygenation is unstable. Alongside it also provides the pulse/Heart Rate $(H R)$ of the patient. The position sensor consists of 3 axis accelerometer to record the body position of the patient such as standing, sitting, supine, prone, left and right.

These sensors are used to help on-site paramedic staff as well as remote doctors in understanding the patient health metrics and tagging the patients that have serious causalities. The tagging information and other useful metrics are displayed on the LCD along with GPS coordinates for patient localization. DigiAID has a keypad interface for the patient to type and show his consciousness/Mental Status $(M S)$ regarding the sensory perception. The values from the keypad are displayed on the LCD. The overall system setup is shown in Fig. 2 and the 


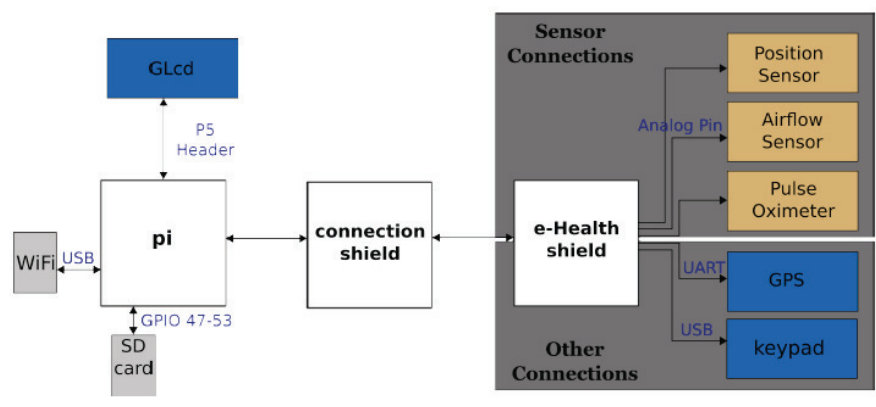

Fig. 2: Overview on the DigiAID system

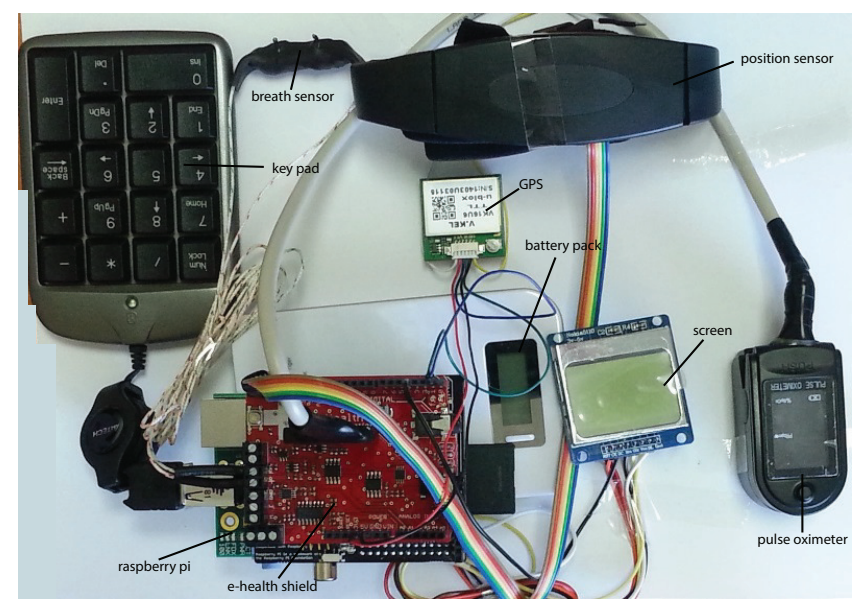

Fig. 3: DigiAID hardware components

complete hardware solution is shown in Fig. 3. The automation of the $M S$ capture is an ongoing step. We primarily plan to use a microphone along with a 3D accelerometer to detect the $M S$ of the patient.

3) Software Design and Implementation: The operating system selected for the Raspberry-Pi board is Raspbian [22], a variant of Debian wheezy, optimized for Raspberry-Pi. It uses the Linux kernel version 3.1. The programming language for development is $\mathrm{C}++$. The final executable will be able to run on the Raspberry-Pi.

The DigiAID software implementation primarily consists of three parts: 1) Initialization, where the status of the attached devices is read and the initialization of sensors is carried out, 2) main Loop, where the core functionality, including the reading of sensor values and their processing according to proposed algorithms, is performed, and 3) finalization, where the reinitialization of the sensors and the logging of key parameters is executed by flushing the buffers onto the SD card.

\section{EVALUATION}

\section{A. Experimental Settings}

In order to evaluate the proposed algorithms, initially in this paper, we consider only daily activities of a healthy person, where generally the output of the system should be "Green" Tag. We are aware of the shortcomings of this assumption, however, due to the complexity of accessing real data or operating on real patients, we are leaving this activity for future work.

The experimental setup encompasses two basic activities of daily routine, i.e., office work (mainly sitting and working on computer), and driving (in urban area). For experiments the Raspberry-Pi with e-health shield, having pulse-oximeter and airflow sensor, are placed on the human body to sense the physiological parameters, i.e., $H R$ and $R R$. The third parameter, i.e., consciousness or $M S$ is assumed to remain TRUE during these activities.

For each activity the person wears the BSN for $30 \mathrm{~min}$. During this period relevant parameter values and the output of the varied proposed algorithms are logged in.

\section{B. Preliminary Experimental Results}

Fig. 4 shows the achieved result. Fig. 4(a) and Fig. 4(b) show the respiratory rate and heart rate of the person during office and driving scenario. The captured $R R$ is low compared to the normal person's average $R R$. After zooming in the results, we found out that the subject person was wearing the airflow sensor upside down. It was also revealed that the wired system was major hurdle and this fact is revealed in Fig. 4(a) where during driving initially the RR is higher and as the time passes due to movement of the person the sensor was slightly misplaced resulting in degraded results. Similar observation was found for the office environment too.

Fig. 4(c) shows that our proposed table driven approach correctly identified the collected parameters and appropriately allocated the criticality class. Due to low $R R$, Class 9 is selected, which corresponds to "Yellow" Tag of the triage system as discussed in [4]. From Fig. 4(d) and Fig. 4(e) it is observed that Fuzzy logic based approach is more accurate and correctly identified the missed values from the sensors. On the other hand, the aggregation based approach as a whole identifies the correct criticality class.

\section{CONCLUSION}

We presented DigiAID, a new open and cost-effective experimental platform that enables for the first time to automatically compute the criticality of Human health conditions and map it to a standard digital tag. Our approach benefits from the ongoing fast progress in body sensor network technologies. DigiAID is a fundamental tool to optimize the rescue operations by reducing the diagnosis time, which may significantly impact the success rate of saving lifes, especially in areas of low medical coverage such as rural or mass casualty incident areas. We showed the viability of our experimental platform through an envelope of real measurements during daily activities.

In future, we aim at conducting experiments that cover critical health situations, which can be effectively achieved using simulated patients. Given that field trials on real patients are hard to achieve, we are cooperating with first response groups to gain access to real patient data to validate our experimental platform. In addition, we aim at coping with inaccuracies in measurements due to either sensor or sensor placement errors as learnt form our first set of experiments. 


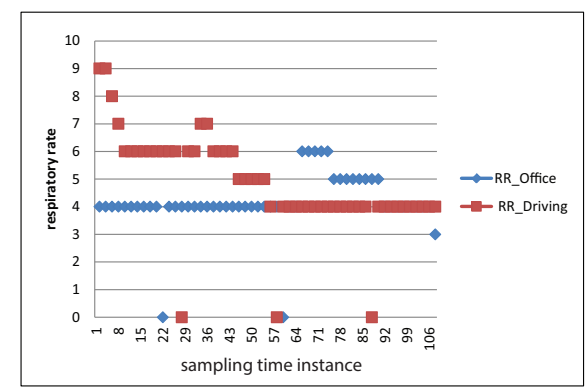

(a) Respiratory rate

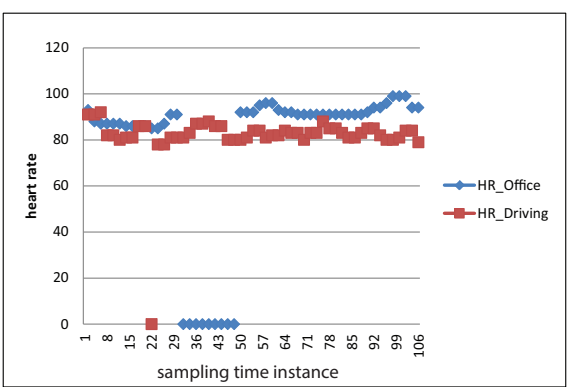

(b) Heart rate

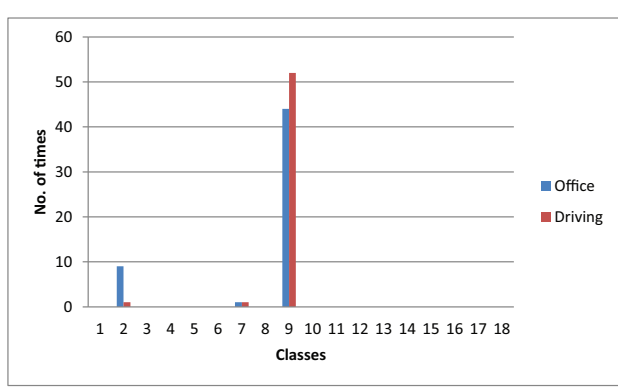

(c) Table based approach

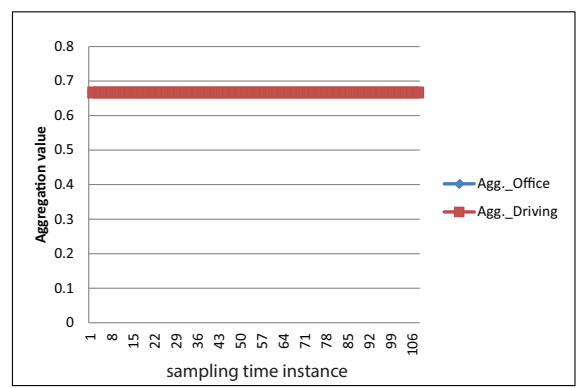

(d) Aggregation based approach

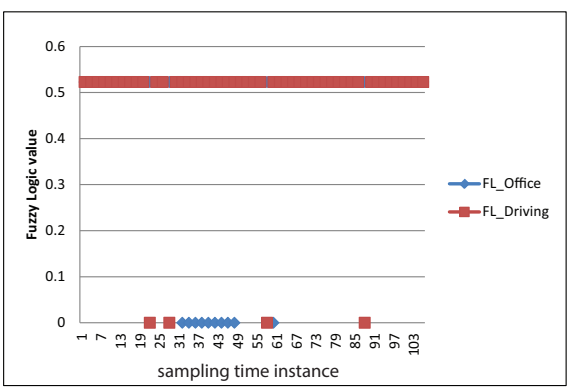

(e) Fuzzy Logic (FL) based approach

Fig. 4: Measurements during office and driving activities

\section{ACKNOWLEDGEMENT}

This work is funded by grant number 10-INF1236-10 from the Long-Term National Plan for Science, Technology and Innovation (LT-NPSTI), the King Abdul-Aziz City for Science and Technology (KACST), Kingdom of Saudi Arabia. We thank the Science and Technology Unit at Umm AlQura University for their continued logistics support. Faisal $\mathrm{K}$. Shaikh is also partially supported by MUET, Pakistan.

\section{REFERENCES}

[1] V. Jones, V. Gay, and P. Leijdekkers, "Body sensor networks for mobile health monitoring: Experience in europe and australia," in Proc. of the Fourth Intl. Conf. on Digital Society (ICDS), 2010, pp. 204-209.

[2] T. Gao et al., "Wireless medical sensor networks in emergency response: Implementation and pilot results," in In Proc. 2008 IEEE International Conference on Technologies for Homeland Security, 2008.

[3] M. Benson, K. L. Koenig, and C. H. Schultz, "Disaster triage: START, then SAVE A new method of dynamic triage for victims of a catastrophic earthquake," Prehospital Disaster Med., pp. 117-124, 1996.

[4] F. Ullah, A. Khelil, A.A. Sheikh, E. Felemban and H. Bojan, "Towards Automated Self-tagging in Emergency Health Cases," in Proc. of The 15th IEEE International Conference on e-Health Networking, Application and Services (Healthcom), 2013.

[5] A. Khelil, "Pa2Pa: Patient to Patient Communication for Emergency Response Support," in Proc. of The 13th IEEE International Conference on e-Health Networking, Application and Services (Healthcom), 2011.

[6] "The CodeBlue Project," in http://fiji.eecs.harvard.edu/CodeBlue.

[7] "MIThril," in www.media.mit.edu/wearables/mithril/.

[8] A.T. Barth et al., "BTEMPO 3.1: A body area sensor network platform for continuous movement assessment," in Proc. Intl. Workshop Body Sensor Networks, 2009, pp. 71-76.

[9] A. Burns et al., "Shimmer: A wireless sensor platform for noninvasive biomedical research," IEEE Sensors Journal, vol. 10, no. 9, pp. 1527$1534,2010$.
[10] Libelium, "e-Health Sensor Platform V2.0 for Arduino and Raspberry $\mathrm{Pi}$," in www.cooking-hacks.com/shop/arduino/starter-kits/ehealthsensors-complete-kit-biometric-medical-arduino-raspberry-pi (Last accessed: 27-07-2014), 2012.

[11] K. Lorincz et al., "Mercury: A Wearable Sensor Network Platform for High-fidelity Motion Analysis," in The 7th ACM Conference on Embedded Networked Sensor Systems (SenSys), 2009.

[12] T. Gao et al., "The Advanced Health and Disaster Aid Network: A Light-weight Wireless Medical System for Triage," IEEE Transactions on Biomedical Circuits and Systems, vol. 1, no. 3, 2007.

[13] V. Gay and P. Leijdekkers, "A Health Monitoring System Using Smart Phones and Wearable Sensors," Special Issue on 'Smart Sensors in Smart Homes' International Journal of Assistive Robotics and Mechatronics (IJARM), vol. 8, no. 2, 2007.

[14] V.M. Jones et al., "Biosignal and Context Monitoring: Distributed Multimedia Applications of Body Area Networks in Healthcare," in Proc. of the IEEE 10th Workshop on Multimedia Signal Processing (MMSP), 2008.

[15] E. Mitchell, S. Coyle, N. E. OConnor, D. Diamond, and T. Ward, "Breathing Feedback System with Wearable Textile Sensors," in Proc. Intl. Conf. Body Sensor Networks, 2010, pp. 56-61.

[16] V.M. Jones et al., "MobiHealth: Mobile Services for Health Professionals," in Book: M-Health: Emerging Mobile Health Systems, 2006.

[17] _ - "Healthcare PANs: Personal Area Networks for trauma care and homecare," in Proc. of Fourth International Symposium on Wireless Personal Multimedia Communications (WPMC), 2001.

[18] P. Leijdekkers and V. Gay, "A Self-Test to Detect a Heart Attack Using a Mobile Phone and Wearable Sensors," in 21st IEEE International Symposium on Computer-Based Medical Systems, 2008, pp. 93-98.

[19] Intel, "The Shimmer Platform," in www.shimmer-research.com.

[20] Samsung, "SIMBAND: A Wearable Sensor Platform for All," in www.samsung.com/us/globalinnovation/innovation areas/\#simband, (Lastaccessed : $27-07-2014), 2014$.

[21] Raspberry Pi Foundation, "Raspberry Pi," in www.raspberrypi.org (Last accessed: 26-06-2014).

[22] The Debian Project, "Raspbian Linux," in www. raspberrypi.org/downloads (Last accessed: 26-06-2014). 